ARTICLE

Received 9 May 2013 | Accepted 24 Oct 2013 | Published 15 Nov $2013 \quad$ DOl: 10.1038/ncomms3818

\title{
Giant infrared absorption bands of electrons and holes in conjugated molecules
}

Matibur Zamadar', Sadayuki Asaoka1, David C. Grills ${ }^{1}$ \& John R. Miller ${ }^{1}$

Infrared (IR) absorption bands often convey identifying information about molecules, but are usually weak, having molar absorption coefficients $<200 \mathrm{M}^{-1} \mathrm{~cm}^{-1}$. Here we report observation of radical anions and cations of conjugated oligomers and polymers of fluorene and thiophene that possess intense mid-infrared absorption coefficients as large as $50,000 \mathrm{M}^{-1} \mathrm{~cm}^{-1}$, perhaps the largest known for molecular species. For anions of fluorene oligomers, $F_{n}, n=2-4$, IR intensities increase almost linearly with $n$, but with a slope much larger than one, indicating that the absorptions are not extensive properties. Large intensities seem to arise from a mechanism known for charged solitons and polarons in conjugated polymers. In this mechanism, vibrations of ungerade symmetry drive substantial displacements of charge, creating the large dipole derivatives responsible for intense IR absorption. Both experiments and calculations find that pairing with counter-ions attenuates IR band intensities. The IR bands may be diagnostic for bound ion pairs and their escape to form free ions.

\footnotetext{
${ }^{1}$ Chemistry Department, Building 555, Brookhaven National Laboratory, Upton, New York 11973-5000, USA. Correspondence and requests for materials should be addressed to J.R.M. (email: jrmiller@bnl.gov).
} 
nfrared (IR) absorption by molecular vibrations can provide diagnostic structural and electronic information about molecules, but it is often weak; absorption coefficients, $\varepsilon$, in the mid-IR of $>200 \mathrm{M}^{-1} \mathrm{~cm}^{-1}$ are considered very strong. Stronger absorptions to $\varepsilon=35,000 \mathrm{M}^{-1} \mathrm{~cm}^{-1}$ are found for the $\mathrm{C} \equiv \mathrm{O}$ stretching bands of metal hexacarbonyls ${ }^{1-3}$. These concur with the adage that bonds with substantial dipole moments tend to give strong IR absorption. Although such strong absorptions are rare in less polar bonds, Sakamoto et al. $^{4}$ reported IR absorption coefficients of $3,450 \mathrm{M}^{-1} \mathrm{~cm}^{-1}$ for the radical anion of $p$-terphenyl and $7,820 \mathrm{M}^{-1} \mathrm{~cm}^{-1}$ for the dianion. These do not come from vibrations of highly polar bonds. Similarly, early research on holes and electrons in conjugated polymers discovered vibrational absorption by 'IR active' molecular vibrations ${ }^{5-9}$. Extensive research on these 'IRAV' bands and Raman scattering in solid films of polymers and their ions ${ }^{10-17}$ produced insight on the natures of the species and fascinating behaviour including possibly large intensities and Fano-like interferences. These are plausibly strong, but absolute intensities were not determined. The IRAV bands were interpreted with an amplitude theory ${ }^{18,19}$ that has been further developed ${ }^{20}$ along with related theories ${ }^{21-23}$ including descriptions with a molecular viewpoint ${ }^{24,25}$. The present work takes advantage of ready determination of absolute IR absorption (extinction) coefficients in solution to find coefficients 10 times larger than that of Sakamoto et al. ${ }^{4}$

\section{Results}

IR spectra of anions. Figure 1 displays IR spectra of radical anions of four fluorene oligomers and a polymer. In each case, the spectrum of the pure solvent (see Supplementary Figs S1 and S2 for solvent spectra) was subtracted from those of the samples containing the anions. Each of these molecules was reduced to make anions using $\mathrm{NaK}$ in deutereo tetrahydrofuran (THF- $\left.d_{8}\right) . \mathrm{F}_{4}$ was also reduced in two other conditions. One was reduction by $\mathrm{Na}$ metal and the other was reduction by $\mathrm{NaK}$ in $90 \%$ THF- $d_{8} /$ $10 \%$ benzene- $d_{6}$. The spectrum with $10 \%$ benzene showed similar frequencies but substantially smaller absorption coefficients. In a similar solution with $20 \%$ benzene- $d_{6}$ (not shown), both frequencies and absorption coefficients were similar to those in $10 \%$ benzene. An expanded version of the $\mathrm{F}_{4}$ anion graph is given in Supplementary Fig. S3. The first five panels of Fig. 1 show midIR spectra from 1,200 to $1,700 \mathrm{~cm}^{-1}$; the sixth shows spectra of all the anions from 2,350 to $4,000 \mathrm{~cm}^{-1}$. Strong solvent absorption precluded determination of spectra of the anions for frequencies $<1,200 \mathrm{~cm}^{-1}$ and from 2,000 to $2,200 \mathrm{~cm}^{-1}$. The region from 1,700 to $2,000 \mathrm{~cm}^{-1}$ is not shown because there was negligible absorption from the anions in the region. Concentrations of the anions were determined to be $0.27,0.11,0.4,0.14$ and $0.18 \mathrm{mM}$ for $\mathrm{F}_{2}, \mathrm{~F}_{3}, \mathrm{~F}_{4}, \mathrm{~F}_{8}$ and $\mathrm{pF}_{30}$, respectively, based on measurements of visible bands (Supplementary Fig. S4) in the solutions of anions and the known absorption coefficients of the anions ${ }^{26,27}$ where determinations of absolute absorption coefficients were aided by the use of pulse radiolysis ${ }^{26}$.
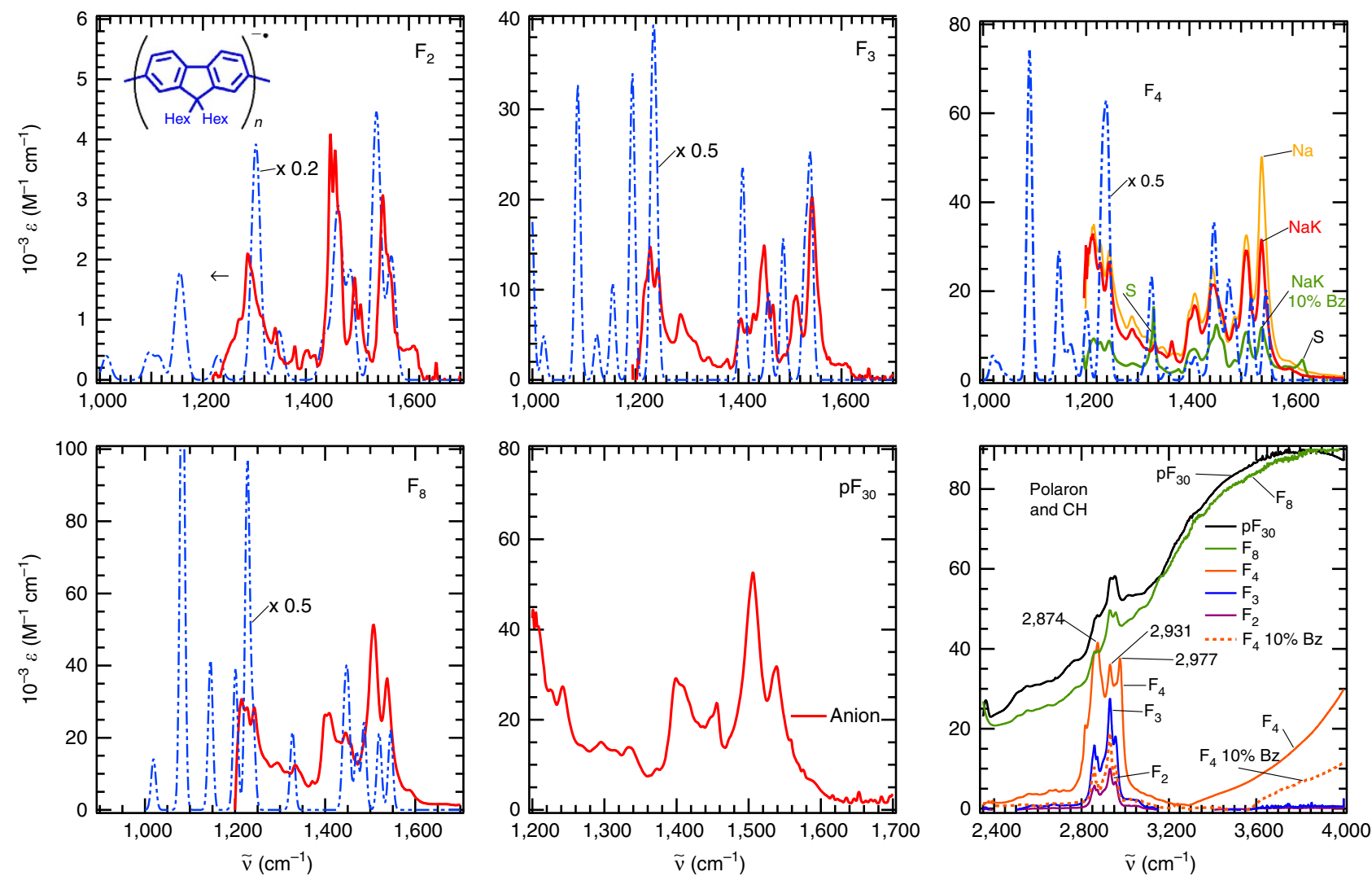

Figure 1 | IR absorption spectra of radical anions. IR spectra of oligofluorenes and a polyfluorene (Hex $=n$-hexyl) with an average length of 30 repeat units in THF- $d_{8}$; the panels are labelled by the molecules from which the anions are formed. $F_{4}$ anions were produced under three conditions (see text). The experimental spectra are compared with computed spectra for ion pairs at $5 \AA$ from the centre of the oligomer (blue dash-dot lines). The calculations give integrated intensities, $A,\left(\mathrm{~km} \mathrm{~mol}^{-1}\right)$. Each computed intensity has been replaced by a Gaussian of $20 \mathrm{~cm}^{-1} \mathrm{full}$ width at half maximum to estimate computed absorption coefficients, using the relation $\varepsilon=4.72 \mathrm{~A}$ (see text). The computed intensities are scaled by factors indicated on the graphs. Bands labelled as $\mathrm{S}$ in the $\mathrm{F}_{4}$ panel are imperfectly subtracted solvent absorptions. Incompletely subtracted solvent absorptions in the spectrum of $\mathrm{F}_{8}^{-}$in the last panel were corrected (see Supplementary Fig. S5). 

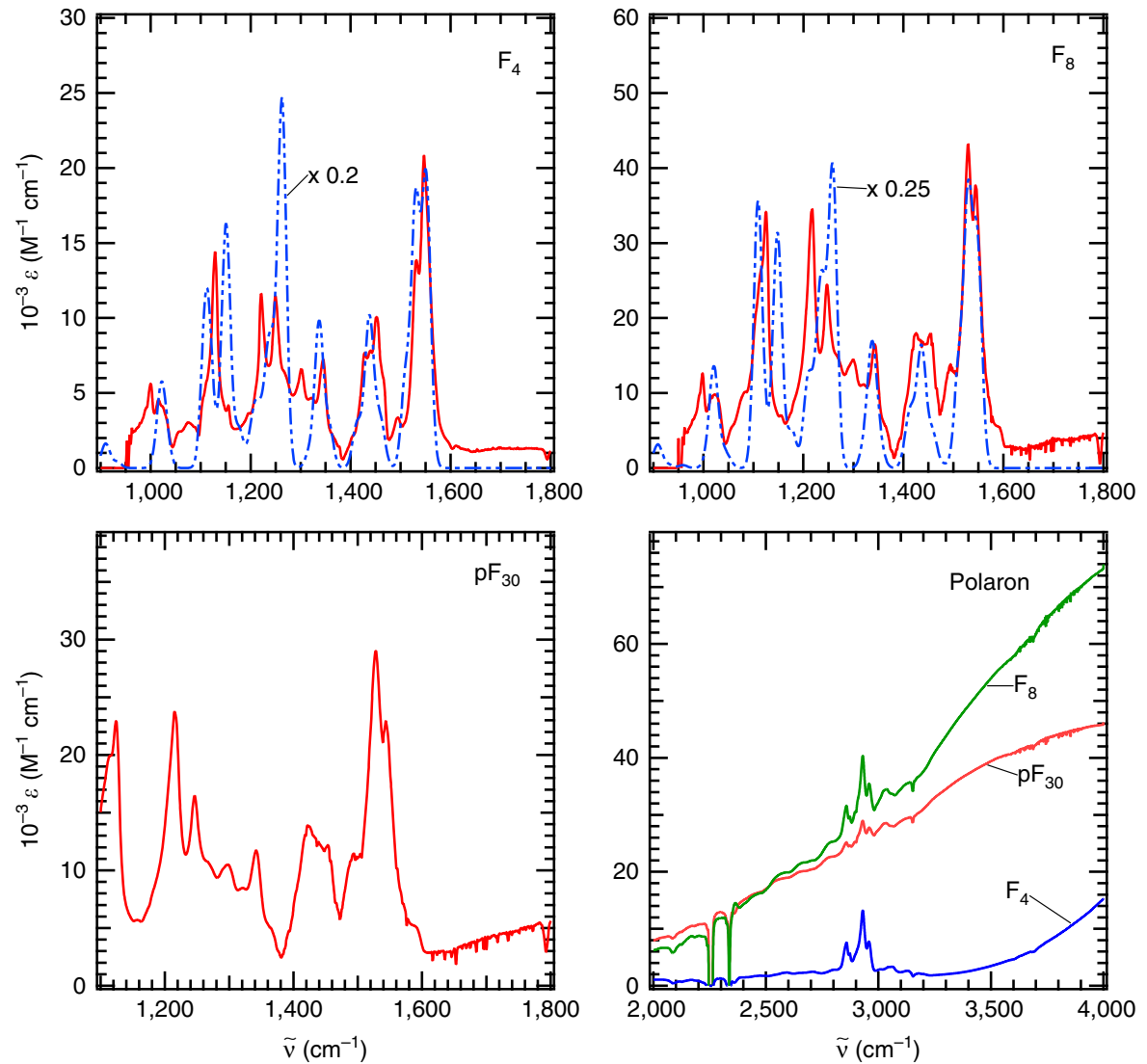

Figure 2 | IR spectra of positive ions. IR absorption spectra of radical cations created by $\mathrm{NOPF}_{6}$ oxidation of $\mathrm{F}_{4}, \mathrm{~F}_{8}$ and $\mathrm{pF}_{30}$ in $\mathrm{CDCl}_{3}$. Other experimental conditions are similar to those in Fig. 1 as are the calculations and their presentation (blue dash-dot lines). The calculations use $\mathrm{PCM}\left(\mathrm{CHCl}_{3}\right)$ with $\mathrm{PF}_{6}^{-}$counter-ions at $5 \AA$.

The experimental spectra are compared with spectra computed by frequency calculations on the geometry-optimized anions in a polarized continuum model (PCM) reaction field simulating THF. The calculations used the $\omega \mathrm{PBE}(\omega=0.1)$ functional that was found to give a good description of the optical transitions of anions of fluorene oligomers and the spatial extent of their polarons, which typically spread over four fluorene repeat units ${ }^{26}$. Computed IR bands in the $\mathrm{CH}$ stretch region (not shown) are small, and will be discussed below. Many or most of the anions are probably ion paired with $\mathrm{K}^{+}$, or possibly $\mathrm{Na}^{+}$counter-ions. Therefore, spectra were computed for ion pairs with $\mathrm{Na}^{+}$. Optimization of the positions of the $\mathrm{Na}^{+}$placed the $\mathrm{Na}^{+}$ unreasonably close to the anions based on the computed optical spectra and energies. It is known that coordination of $\mathrm{Na}^{+}$or $\mathrm{K}^{+}$by THF causes these ions to pair more weakly with anions probably because the alkali ions surrounded by THF molecules act like much larger ions. The ion-pair calculations therefore optimized and computed IR spectra for anions with $\mathrm{Na}^{+}$ counter-ions held at fixed, $5 \AA$, distances. This distance is between the $\sim 2.5-\AA$ distance of closest approach from optimizations and the $7-8-\AA$ distance estimated for solventseparated ions pairs ${ }^{28}$. As the actual distance is not known, these ion-pair calculations are provisional and are meant to illustrate trends. An example structure is shown in Supplementary Fig. S6.

The relation $A\left(\mathrm{~km} \mathrm{~mol}^{-1}\right)=0.0106 \varepsilon w$, where $w$ is the full width at half maximum (FWHM) of the band in $\mathrm{cm}^{-1}$ is used in Fig. 1 to compare the computed integrated intensities $A$ to observed spectra. This relation assumes that the bands are approximately Gaussian in shape. As bands often overlap, their widths are not precisely determined, but many of the widths appear to be $\sim 20 \mathrm{~cm}^{-1}$, giving $\varepsilon=4.72 \mathrm{~A}$. With this relation, the computed intensities were larger than experimental intensities.

IR spectra of cations. IR spectra of $\mathrm{F}_{4}, \mathrm{~F}_{8}$ and $\mathrm{pF}_{30}$ produced by oxidation by nitrosonium hexafluorophosphate $\left(\mathrm{NOPF}_{6}\right)$ are shown in Fig. 2. The $\mathrm{CDCl}_{3}$ solvent permitted acquisition of spectra of the cations over a slightly larger frequency range. Absorption coefficients for the $\mathrm{pF}_{30}$ cation were calibrated using $\varepsilon\left(\mathrm{pF}^{+} \bullet\right)$ reported by Takeda et al. ${ }^{27}$ For $\mathrm{F}_{4}$ and $\mathrm{F}_{8}$ cations, absorption coefficients of the visible bands were assumed to be similar to those of the corresponding anions ${ }^{26}$. We estimate that this approximation raises the uncertainty of the IR absorption coefficients of the cations to $\pm 30 \%$. The IR spectra of ion-paired cations used $\mathrm{PF}_{6}^{-}$counter-ions.

Figure 3 shows IR spectra of the cation of the polythiophene, poly(3-decylthiophene-2,5-diyl) (P3DT). P3DT ${ }^{+} \bullet$ was created by similar methods in $\mathrm{CDCl}_{3}$. Calibration of absorption coefficients used the result of Takeda and Miller ${ }^{29}$. In Fig. 3, we see that the maximum of the polaron band is at $\sim 3,000 \mathrm{~cm}^{-1}$ and that the band overlaps even the mid-IR vibrations from 1,000 to $1,400 \mathrm{~cm}^{-1}$, which are broader than those of the fluorenes.

\section{Discussion}

The main features of the spectra of the ions are described here and discussed below after noting relations to known information: 


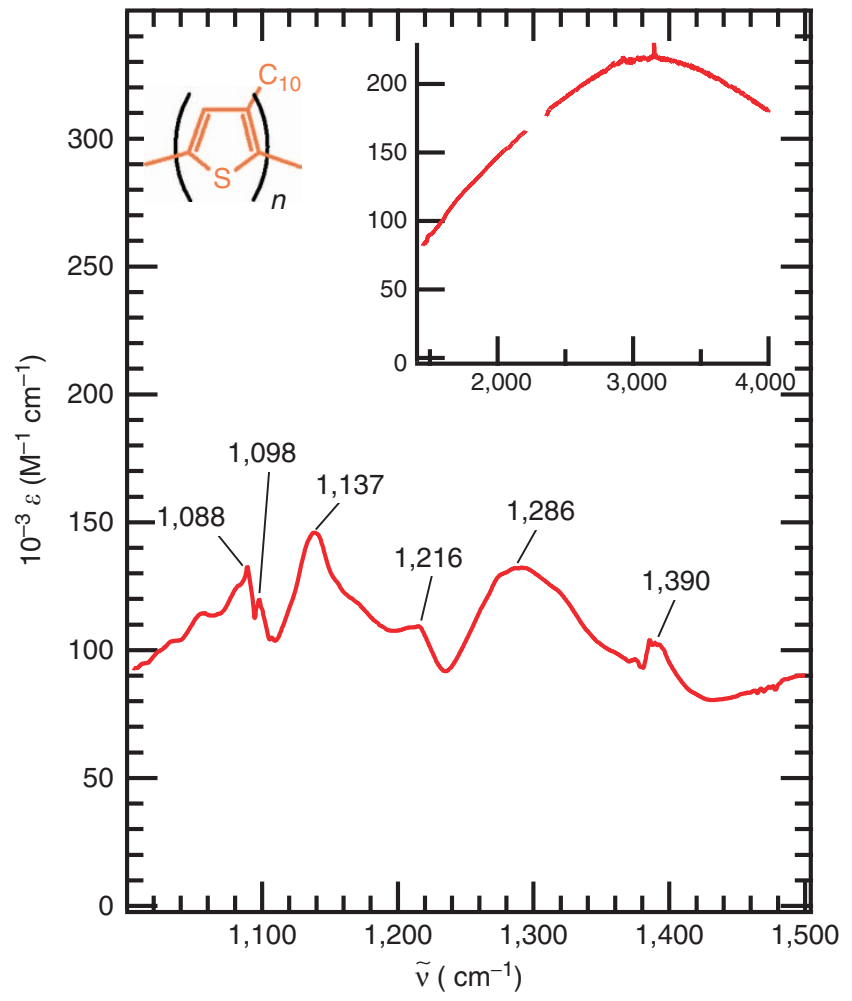

Figure 3 | IR spectra of a polythiophene cation. The IR absorption spectrum of P3DT ${ }^{+}$in $\mathrm{CDCl}_{3}$ under conditions as in those of Fig. 2.

intensities of the main IR bands, expressed as molar absorption coefficients, are large, perhaps the largest we know of for any molecular species. The intensities for oligofluorene ions in Figs 1 and 2 depend strongly on length as seen in the plot of peak intensity in Fig. 4 and in Supplementary Fig. S7, which plots intensities (extinction coefficients) integrated over four regions to sense averages over congested regions. Although this increase looks approximately linear from $\mathrm{F}_{2}$ to $\mathrm{F}_{4}$, the slope is not unity: as the length doubles, the intensities of the bands shown increase by an average of a factor $>7$. The intensities level off for oligomers longer than $\mathrm{F}_{4}$, except for the $\mathrm{CH}$ stretch region, where intensities decrease. At high frequencies, usually $\sim 2,000 \mathrm{~cm}^{-1}$, broad continuous absorptions appear because of polaron bands that are well known for holes and electrons in conjugated polymers and oligomers. For the molecules studied here, the polaron bands were reported earlier in the near $\mathrm{IR}^{26,27,29}$. To estimate the IR intensities of $\mathrm{CH}$ stretches in Fig. 4 and Supplementary Fig. S7, the absorption due to the polaron bands was subtracted using linear interpolation. Intensities of $\mathrm{F}_{4}^{-} \bullet$ decreased by more than a factor of two on addition of a non-polar solvent, $10 \%$ benzene- $d_{6}$.

IR spectroscopy has been shown to provide information about the nature of charge carriers in conjugated polymers ${ }^{30}$, and as discussed above, early research on holes and electrons in conjugated polymers discovered absorption by 'IR active, IRAV' molecular vibrations ${ }^{5-9}$. Although the absolute intensities of these IRAV bands were not determined, it is plausible that the IRAV bands have absolute intensities similar to those described here.

The intensities found here are astonishing, raising the question why are they so strong? Theories of IR intensities ${ }^{31-34}$ have evolved $^{23-25}$ to include contributions from two principal factors: dipole derivatives and proximity to electronic transitions. Substantial dipole derivatives arise when charge distributions evolve in response to vibrations ${ }^{35,36}$ and these can become 'massive', for example, for charged solitons in polyacetylene ${ }^{37,38}$.

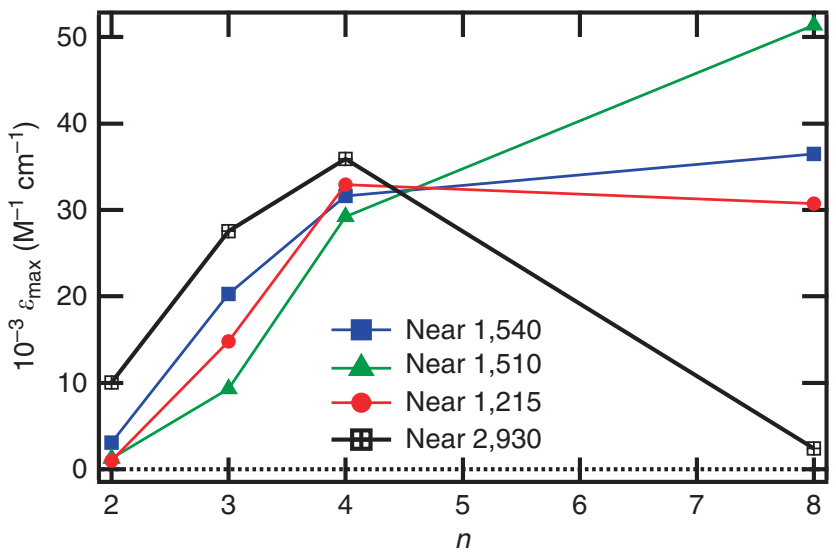

Figure 4 | Effect of length. Intensities at the peaks of four principal IR transitions in radical anions of fluorene oligomers, $F_{n}$, as a function of their lengths, $n$, in repeat units from experimental data in Fig. 1. The actual peak positions vary slightly with the length of the oligomer. For example, the positions of the peak labelled as near 1,540 are at 1,549; 1,543; 1,540 and $1,538 \mathrm{~cm}^{-1}$ for $n=2,3,4$ and 8 . Computed spectra were in rough agreement with observed spectra, but for the most intense bands the computed frequencies often vary by up to $20 \%$ from the most intense observed frequencies. Agreement of computed frequencies with observed frequencies is best for $\mathrm{F}_{2}^{-}$and becomes poorer for longer oligomers.

The second contribution, to be discussed in connection with $\mathrm{CH}$-stretching vibrations, arises from vibronic interactions with low-lying electronic transitions that enhance the dipole derivatives. Both factors are unusually effective in the conjugated ions studied here. We describe below how large dipole derivatives are enhanced for the $\mathrm{C}=\mathrm{C}$ stretches and $\mathrm{CH}$ bends seen from 1,000 to $1,700 \mathrm{~cm}^{-1}$, while interactions with electronic excitations seem to enhance $\mathrm{CH}$-stretching intensities.

For several of the vibrational modes in the $1,000-1,700 \mathrm{~cm}^{-1}$ region, vibrational displacement of the nuclei provokes a larger displacement of the polaron. This is seen in Fig. 5, which displays the Kohn-Sham singly occupied molecular orbital (SOMO) computed for $\mathrm{F}_{5}^{-} \bullet$. The top panel shows the SOMO of the optimized (undisplaced) structure. The next panel shows the SOMO for structures stretched or compressed to the classical turning points of the $945 \mathrm{~cm}^{-1}$ vibration, where we observe a notable displacement of the SOMO bearing the charge. The stretch or compression of these structures produces dipole moments of 9.9 Debye in opposite directions: the charged polaron vibrates along with the molecular vibration producing big changes in dipole moment. This motion is more obvious in the large displacements of the SOMO with stretching or compression to five times the classical turning point, displayed in the lower panel of Fig. 5. The mechanism giving 'massive' charge displacements for solitons ${ }^{37,38}$ and for polarons ${ }^{20-22,39}$ is pictorially described in Fig. 5, and may resemble 'the changes in electronic structures induced by specific vibrations' mentioned by Sakamoto et al. ${ }^{4}$

Figure 5 gives us a picture of polarons as objects that are not static. Instead, the polaron vibrates synchronously with several molecular vibrations. As for charged solitons ${ }^{37,38}$, an important feature to produce large IR intensities for the $945 \mathrm{~cm}^{-1}$ mode, discussed in Fig. 5, and other bands giving large IR intensities, (for example, 1,$038 ; 1,048 ; 1,142 ; 1,171 ; 1,217 ; 1,403 ; 1,487$; and $1,517 \mathrm{~cm}^{-1}$ ) is that these vibrations are antisymmetric, transforming with the $\mathrm{B}$ representation in the $\mathrm{C} 2$ structure of $\mathrm{F}_{5}^{-} \bullet$. Supplementary Fig. S8 shows vector diagrams for displacements in these modes. Each of these modes that give high 
0.0
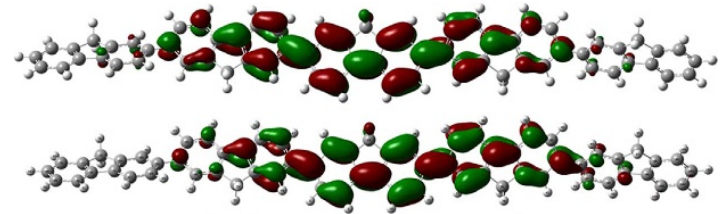

1.0
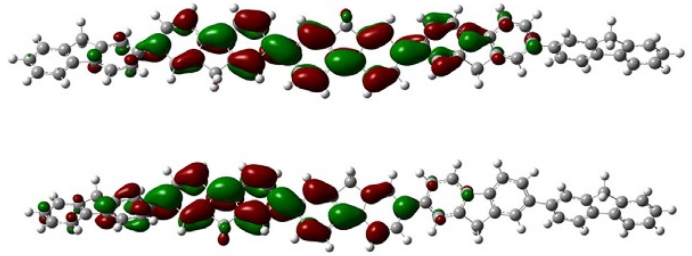

5.0

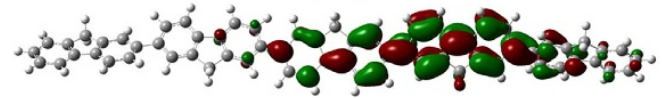

Figure 5 | Shift of charge on vibrational displacement. Kohn-Sham SOMO orbitals (3-21G, $\omega$ PBE $\omega=0.1$ in PCM for THF) in $\mathrm{F}_{5}^{-} \bullet$. At the top, labelled 0 , is the SOMO of optimized $\mathrm{F}_{5}^{-} \bullet$. Next, labelled 1, are SOMOs from single-point calculations on structures stretched and compressed to the classical turning points of the $945 \mathrm{~cm}^{-1}$ vibration. At the bottom, labelled 5, are SOMOs in similar structures displaced to five times the classical turning points.

IR intensity contains displacements distributed along the length of the polaron. These modes comprise $\mathrm{CH}$ rocks and $\mathrm{CC}$ stretches along the axis of the molecule, so their displacements produce net displacement of the charge of the polaron. As for charged solitons ${ }^{37,38}$, they are ungerade: stretching of a bond on one side of the molecule is synchronous with compression of the symmetrically related bond on the opposite side of the molecule. The computed integrated intensity of the $945-\mathrm{cm}^{-1}$ mode is $A=131,877 \mathrm{~km} \mathrm{~mol}^{-1}$. A somewhat similar, but symmetric (A) vibration at $995 \mathrm{~cm}^{-1}$ has an integrated intensity of only $1.3 \mathrm{~km} \mathrm{~mol}^{-1}$. For such symmetric vibrations, charge on the left and right sides of the molecule both move towards the centre at the same time, so the polaron vibrates, but with no net change of dipole moment, and hence negligible IR intensity. For vibrations of appropriate symmetry, the frequency calculation in Gaussian 09, or other computational programs, captures the large dipole derivatives generated by this mechanism, yielding the large computed intensities.

Exceptional dipole derivatives thus arise because the charge, self-localized in a polaron as illustrated in Fig. 5, moves in response to a vibration with appropriate symmetry, generating a strong dependence of dipole moment on vibrational displacement. This effect is large when uncharged regions are present to the left and right of the polaron. Uncharged regions are seen at both ends in Fig. 5, top panel. For ions of $\mathrm{F}_{2}$ and $\mathrm{F}_{3}$, the polarons are confined by the short length of the oligomers, so less displacement of the polaronic charge occurs on vibration. As polarons in $\mathrm{F}_{n}$ oligomers have a length of $\sim 4.3$ repeat units ${ }^{26,27}$, the IR enhancements are optimal at lengths slightly longer than four repeat units. For this reason, $F_{5}$ was chosen for the illustration in Fig. 5. The mid-IR intensities in Fig. 4 increase approximately linearly with length for $\mathrm{F}_{n}^{-} \cdot(n=2-4)$. This increase begins at an intercept of zero intensity at $n=1.9 \pm 0.1$ repeat units ( 3.8 benzene rings). Thus, for molecules with lengths of $<3.8$ benzene rings, the intensities are small, but they increase rapidly above this length.

The observed IR spectra of $\mathrm{F}_{4}^{-\bullet}$ in Fig. 1 were strongest when $\mathrm{F}_{4}$ was reduced by $\mathrm{Na}$, weaker when reduction was by $\mathrm{NaK}$ and still weaker when $10 \%$ benzene- $d_{6}$ was added. $\mathrm{As} \mathrm{Na}^{+}$is more strongly solvated ${ }^{40}$ by THF than is $\mathrm{K}^{+}$, this order is consistent with weaker IR bands in $\left(\mathrm{M}^{+}, \mathrm{F}_{4}^{-\bullet}\right)$ when the ion pairing is stronger. Computed IR spectra in Supplementary Fig. S9 predict that most IR bands are strongest in the free ion, and become weaker as the counter-ion is closer. Together, the experiments and calculations support the idea that ion pairing tends to decrease IR intensities. The calculations also predict that the positions of some IR bands shift with ion pairing and that some exceptionally large intensities will occur at frequencies below $1,100 \mathrm{~cm}^{-1}$, especially in free ions (see also Supplementary Fig. S9). These calculated results are not tested for the anions in the present experiments because of solvent absorption. However, the region was observable for cations in $\mathrm{CDCl}_{3}$ because of the good transparency of this solvent, and the observed absorption coefficients were not larger than those in other regions. The intensities were consistent with those computed for ion pairs (see Fig. 2). With the low dielectric constant $(\varepsilon=4.89)$ of chloroform, it is plausible that there are few free ions, and that the ion pairs are tightly bound.

Quantum-chemical calculations often give good predictions of IR spectra for small molecules, including ions of terphenyl ${ }^{4}$, and even for charge-transfer salts ${ }^{41}$. The calculations reproduce some features of the observed spectra fairly well. However, they fail to predict bands observed near $1,300 \mathrm{~cm}^{-1}$ for $\mathrm{F}_{3}^{-\bullet}$ and some predicted bands are too large, whereas others are too small. The calculations are somewhat better for fluorene cations in Fig. 2. The failure of the calculations to give excellent descriptions of the observed spectra may arise because they are on optimized structures, each with a single conformation. The actual molecules almost certainly undergo conformational fluctuations, especially of dihedral angles between adjacent repeat units. The calculations do predict intensities to increase by factors of $\sim 2$ from $\mathrm{F}_{2}^{-}$to $\mathrm{F}_{3}^{-\bullet}$, but actual increases are much larger (see Fig. 4). This may signal that the range correction of the $\omega \mathrm{PBE}$ functional does not fully predict the confinement of the charge in short oligomers. However, it may mean that ion pairing is tighter to the shorter $\mathrm{F}_{2}^{-} \bullet$, producing lower intensities; the calculations used a constant ion-pairing distance. The calculations also locate counter-ions $5 \AA$ from the centre of the molecules, whereas the actual positions certainly fluctuate, possibly altering vibrational intensities. Supplementary Fig. S11 shows very substantial changes of a computed IR spectrum by movement of the counter ion. An accurate computational rendering is therefore likely to be challenging.

The largest contrast between observed and computed intensities occurs in the $\mathrm{CH}$-stretching region, $2,850-3,200 \mathrm{~cm}^{-1}$. Observed bands reach absorption coefficients of nearly $10,000 \mathrm{M}^{-1} \mathrm{~cm}^{-1}$ for $\mathrm{F}_{2}^{-} \bullet$ and rise to $\sim 40,000 \mathrm{M}^{-1} \mathrm{~cm}^{-1}$ for $\mathrm{F}_{4}^{-} \bullet$. However, in the $\mathrm{CH}$ region the largest computed absorption coefficient for $\mathrm{F}_{4}^{-}$for the free ion is smaller by a factor of eight. For $5 \AA$ ion pairs, the largest computed band is smaller by another factor of two. Supplementary Figs S10 and S11 give examples of the near absence of $\mathrm{CH}$-stretching vibrations in a computed spectrum. This pattern holds for other ions; computed intensities are much smaller than those observed. Supplementary Fig. S12 displays displacement vectors for two of the $\mathrm{CH}$-stretching vibrations giving the largest computed IR intensities. All the $\mathrm{CH}$-stretching vibrations are localized, as are the two in Supplementary Fig. S12, and for most their displacements are in nodal planes of the $\pi$ electrons. Such vibrations are expected to produce only small dipole derivatives, consistent with the computed results.

The fact that large $\mathrm{CH}$ bands are observed despite weak expected and computed dipole derivatives might be understood on the basis of enhanced IR intensities due to vibronic coupling $4,17,20,24,25,39$ to low-energy electronic excited states. Nafie's ${ }^{25}$ description for the transition dipole of an IR band at frequency $\omega_{\mathrm{a}}$ multiplies the contributions of dipole 
derivatives to transition moments by a term

$$
L L_{\mathrm{e}}=\left(\omega_{\mathrm{eg}}^{0}\right)^{2} /\left[\left(\omega_{\mathrm{eg}}^{0}\right)^{2}-\left(\omega_{\mathrm{a}}^{2}\right)\right]
$$

$L L_{\mathrm{e}}$ describes contributions from each electronic transition at frequency $\omega_{\mathrm{eg}}^{0}$. When all electronic excitations lie much higher than the vibrational excitation $\left(\omega_{\mathrm{eg}}^{0} \gg \omega_{\mathrm{a}}\right)$, dipole derivatives are amplified little by electronic excitations. It is apparent in Figs 1-3 that the $\mathrm{P}_{1}$ transitions of polarons ${ }^{24,25}$ provide low-lying electronic states at energies near to those of the $\mathrm{CH}$ vibrational transitions that could amplify their IR intensities. The intensities computed here (Figs 1 and 2, Supplementary Figs S9 and S11) for the $\mathrm{CH}$ vibrations are much weaker than the observed $\mathrm{CH}$ bands, possibly because vibronic coupling to excited states is not yet incorporated into Gaussian 09 for calculation of IR intensities. Equation (1) estimates enhancements of 1.7, 1.4 and 1.3 for $3,100 \mathrm{~cm}^{-1}$ bands in anions of $F_{4}, F_{3}$ and $F_{2}$. These small enhancements, based on maxima of the $\mathrm{P}_{1}$ polaron bands, are well below what is needed to understand the large observed $\mathrm{CH}$ bands, but it is conceivable that the fluctuating polarons can give very small denominators in equation (1) and thus much larger enhancements.

Although the polaron band enhances intensities of $\mathrm{CH}$ vibrations, there can be too much of a good thing. In the $\mathrm{CH}$ regions of $\mathrm{F}_{8}^{-} \bullet$ and $\mathrm{pF}_{30}^{-} \bullet$ (Fig. 1) and $\mathrm{F}_{8}^{+} \bullet$ and $\mathrm{pF}_{30}^{+} \bullet$ (Fig. 2) the broad, continuous polaron bands are not just near the $\mathrm{CH}$ vibrations but overlap them. Instead of producing still larger intensities from the $\mathrm{CH}$ vibrations, this overlap with the polaron bands seems to reduce their intensities relative to those in $\mathrm{F}_{4}^{-} \bullet$ or $\mathrm{F}_{4}^{+} \bullet$. This interesting effect could provide clues as to the nature of the broad polaron absorption bands. For P3DT ${ }^{+}$(Fig. 3 ), the polaron absorption extends to such low energies that the overlap and loss of intensity occurs even for the mid-IR vibrations, which appear to be broadened and of lower intensity.

This behaviour and related anti-resonances ${ }^{20}$ can be understood in terms of amplitude theory ${ }^{18,20-22,39}$ or the theory of Nafie ${ }^{25}$, which predicts intensity enhancement from a larger positive dipole matrix element when the low-lying electronic state is above the vibrational excited state $\left(\omega_{\mathrm{eg}}^{0}>\omega_{\mathrm{a}}\right)$ but a negative matrix element when $\omega_{\mathrm{eg}}^{0}<\omega_{\mathrm{a}}$. If both occur simultaneously, interference (cancellation) can occur, but simultaneity is important because, as Nafie notes, the dipole strength is the 'absolute square of the electric-dipole transition moment, always a positive quantity.' Interference between contributions from polaron states above and below the vibrational quantum could explain the lower intensities when the polaron bands overlap the vibrations. This would require that the polaron bands are homogeneously broadened, at least on the timescale of vibration.

In conclusion, extremely intense mid-IR bands were observed to arise from molecular vibrations in conjugated oligomers and polymers. These intense bands occur only in molecules containing electrons or holes (radical anions or cations), not in neutrals. Intensities of these IR bands are competitive with the largest IR absorption coefficients measured for molecular species. They are smaller in short oligomers and increase with length of the oligomer until the oligomer becomes longer than the length of the polaron. The IR absorption bands are similar or identical to 'IR active, IRAV' bands observed in polymer films. Intensities of the IRAV bands have not been reported, but it is likely that they are similar to those determined here. Explanations for the astonishing intensities seem to lie in the properties of polarons, which undergo motions in response to molecular vibrations having appropriate symmetry. For $\mathrm{F}_{5}^{-} \bullet$, these are vibrations transforming with $\mathrm{B}$ representations in $\mathrm{C} 2$ symmetry. These vibrational motions drive the polaron along the long axis of the conjugated molecule generating large dipole moments as discussed earlier for charged solitons and polarons ${ }^{18,20-22,37-39}$. The present findings indicate that polarons in conjugated chains are dynamic, undergoing changes of charge density driven by molecular vibrations.

Density functional theory calculations gave a fair, although not excellent, description of the frequencies and intensities. Experiments and calculations together point to a substantial sensitivity of the IR bands to ion pairing: Measurement of IR bands during charge separation may have the potential to distinguish ion pairs from free ions, possibly even distinguishing intermediates in the charge escape process. The second mechanism producing large IR intensities involves vibronic interaction of vibrations with lowlying electronic states. Here the vibrational transitions borrow intensity from low-energy transitions of polarons.

\section{Methods}

Chemicals. Regioregular P3DT (Rieke Metals, Inc., $>98.5 \%$ head-to-tail) was purchased from Aldrich and used without further purification. Gel permeation chromatography measurements revealed a number-averaged molecular weight $M_{\mathrm{n}} \approx 38,000$ and a weight-averaged molecular weight $M_{\mathrm{w}} \approx 120,000$ g per mole (polydispersity index $\left.M_{\mathrm{w}} / M_{\mathrm{n}} \approx 3.2\right)^{29}$. From the measured $M_{\mathrm{n}}$, the average number of units is estimated to be $n \sim 170$. The syntheses and characterization of oligofluorenes $(\mathrm{F})$ and polyfluorene $\left(\mathrm{pF}_{30}\right)$ were reported previously ${ }^{42}$. The obtained $\mathrm{pF}$ was fractionally separated with preparative scale gel permeation chromatography to provide samples with different average lengths. The polydispersity of the $\mathrm{pF}_{30}$ used here was 1.82 . Sodium potassium alloy from Aldrich and $\mathrm{NOPF}_{6}$ from Alfa-Aesar were used as received.

The molecules under study were reduced or oxidized in deuterated THF or chloroform. These liquids were able to dissolve the molecules and had reasonably good IR transmission. Their spectra and those of some other solvents are shown in Supplementary Figs S1 and S2. THF- $d_{8}$ (Aldrich, anhydrous, inhibitor free) was stirred with sodium potassium alloy for several days, vacuum distilled from sodium potassium alloy and stored under argon. Deutereo chloroform (Aldrich, HPLC grade, stabilized with aluminium metal) was treated repeatedly with $5 \AA$ molecular sieves and stored under argon.

Chemical reduction and oxidations. All reductions and oxidations have been conducted inside an inert atmosphere glovebox. Oligomers $\left(\mathrm{F}_{n}\right)$ and polymers $\left(\mathrm{pF}\right.$ and P3DT) were reduced with sodium potassium alloy $(\mathrm{NaK})$ in THF- $d_{8} . \mathrm{F}_{4}$ was also reduced by $\mathrm{Na}$ metal chunks, but the reaction with $\mathrm{Na}$ metal took a few days to obtain sufficient concentration of anion for IR studies. Oxidations were carried out heterogeneously with $\mathrm{NOPF}_{6}$ in deutereo chloroform. Both reactions were monitored with ultraviolet-visible-near IR in a 1.0 or $0.5-\mathrm{mm}$ path length dry quartz optical cell. Concentrations of anions were determined from the absorptions (Supplementary Fig. S3) along with known absorption coefficients ${ }^{26,27,29}$. Care was taken to add one or less reducing or oxidizing equivalent to each oligomer, or for polymers, to produce polarons well spaced from each other in the polymer chains. Absorption coefficients of $\mathrm{pF}$ anions and cations are similar ${ }^{27}$. For cations of fluorene oligomers, the absorption coefficients are not known but were estimated to be similar to those of the corresponding anions.

IR spectroscopy. IR spectroscopy was carried out in a gastight Harrick Scientific demountable liquid transmission cell (DLC-S25) constructed from a chemically inert stainless steel body. Two ports with Swagelok fittings permit static and flow experiments. The two $\mathrm{CaF}_{2}$ windows used are separated with PTFE spacers; a $0.95-\mathrm{mm}$ spacer was used in the present experiments. For sample preparation, the cell was filled in the glovebox by injecting the reactive cation or anion solution through one port. The cell was rinsed twice with the reduced or oxidized solution; the spectrum was taken from the third filling. The two ports were closed tightly with Swagelok caps and the cell transported in ambient air to a Thermo Scientific Nexus 670 FTIR spectrometer where the IR spectrum was recorded within $10 \mathrm{~min}$. Fourier transform infrared spectra were recorded at $2 \mathrm{~cm}^{-1}$ spectral resolution with the Happ-Genzel apodization function and two levels of zero filling. Spectra of the anions in the sealed cell were typically stable for an hour with the cell surrounded by air, but some possible loss contributed to the uncertainty of the reported absorption coefficients, which we estimate to be $\pm 20 \%$.

Density functional theory computations. Density functional theory computations were carried out with Gaussian 09, C.01 (ref. 43). Frequency calculations were performed after optimization of geometries, both using the $\omega \mathrm{PBE}$ long rangecorrected functional ${ }^{44}$, with $\omega=0.1$, and the PCM reaction field to simulate solvation in either THF or chloroform. Vibrational frequencies were unscaled. The reported results used the $3-21 \mathrm{~g}$ basis set. Tests with $6-31 \mathrm{~g}^{*}$ calculations produced only small differences as did tests with the SMD reaction field. Depictions used GaussView 5. 


\section{References}

1. Abel, E. W. \& Butler, I. S. Intensity of the carbonyl stretching modes in certain halogenocarbonyl derivatives of chromium, tungsten, manganese, rhenium and iron. Trans. Faraday Soc. 63, 45-55 (1967).

2. Ahmed, M. S. \& Elmottaleb, A. Absolute co-stretching intensity and 2pi orbital population on carbonyl groups in 6-coordinate carbonyl transition-metal complexes. J. Mol. Struct. 25, 438-441 (1975)

3. Baibich, I. M., English, A. M. \& Butler, I. S. Absolute integrated Infrared intensities of the carbonyl, thiocarbonyl, and selenocarbonyl stretching modes in the chalcocarbonyl complexes $\mathrm{Cr}(\mathrm{Co}) 5(\mathrm{Co}), \mathrm{Cr}(\mathrm{Co}) 5(\mathrm{Cs}), \mathrm{Cr}(\mathrm{Co}) 5(\mathrm{Cse})$ and (Eta-6-C6h6)Cr(Co)2(Co), (Eta-6-C6h6)Cr(Co)2(Cs), (Eta-6-C6h6)Cr(Co) 2(Cse). Organometallics 3, 1786-1789 (1984).

4. Sakamoto, A., Harada, T. \& Tonega, N. A new approach to the spectral study of unstable radicals and ions in solution by the use of an inert gas glovebox system: observation and analysis of the infrared spectra of the radical anion and dianion of p-terphenyl. J. Phys. Chem. A 112, 1180-1187 (2008).

5. Kim, Y. H., Foster, C. M. \& Heeger, A. J. Polarons in high-Tc superconductors-Irav modes and electronic-transitions to gap states as in conducting polymers. Synth. Met. 29, F603-F608 (1989).

6. Poplawski, J., Ehrenfreund, E., Glenis, S. \& Frank, A. J. Photoexcitation spectroscopy of poly(3-methylthiophene)-electronic bands and vibrationalmodes. Synth. Met. 28, C335-C440 (1989).

7. Halliday, D. A. et al. Synthesis and characterization of doped and undoped poly(2,5-dimethoxyphenylene vinylene). Synth. Met. 41, 931-934 (1991).

8. Park, Y. W. et al. Metallic properties of transition-metal halides doped polyacetylene-the soliton liquid-state. Synth. Met. 41, 27-32 (1991)

9. Voss, K. F. et al. Substitution effects on bipolarons in alkoxy derivatives of poly(1,4-phenylene-vinylene). Phys. Rev. B 43, 5109-5118 (1991).

10. Moses, D., Dogariu, A. \& Heeger, A. J. Ultrafast photoinduced charge generation in conjugated polymers. Chem. Phys. Lett. 316, 356-360 (2000).

11. Miller, E. K. et al. Observation of photoinduced charge transfer in conducting polymer fullerene composites using a high-bandgap polymer. Synth. Met. 84, 631-632 (1997).

12. Khatib, O. et al. Infrared spectroscopy of narrow gap donor-acceptor polymerbased ambipolar transistors. Phys. Rev. B 86, 195109 (2012).

13. Khatib, O. et al. Infrared signatures of high carrier densities induced in semiconducting poly(3-hexylthiophene) by fluorinated organosilane molecules. J. Appl. Phys. 107, 123702 (2010).

14. Neugebauer, H. et al. Electrochemically and photoinduced infrared bands in PPV: a comparative study. Opt. Probes Conj. Polym. 3145, 507-515 (1997).

15. Neugebauer, $H$. Infrared signatures of positive and negative charge carriers in conjugated polymers with low band gaps. J. Electroanal. Chem. 563, 153-159 (2004).

16. Zhuo, J. M. et al. Direct evidence for delocalization of charge carriers at the fermi level in a doped conducting polymer. Phys. Rev. Lett. 100, 186601 (2008).

17. Sheng, C. X., Basel, T., Pandit, B. \& Vardeny, Z. V. Photoexcitation dynamics in polythiophene/fullerene blends for photovoltaic applications. Org. Electron. 13, 1031-1037 (2012).

18. Horovitz, B. Infrared activity of peierls systems and application to polyacetylene. Solid State Commun. 41, 729-734 (1982).

19. Cao, Y., Guo, D., Pang, M. \& Qian, R. Y. Studies on iodine doped thiophene oligomers. Synth. Met, 18, 189-194 (1987)

20. Osterbacka, R. et al. Photoinduced quantum interference antiresonances in pi-conjugated polymers. Phys. Rev. Lett. 88, 226401 (2002).

21. Girlando, A., Painelli, A. \& Soos, Z. G. Electron-phonon coupling in conjugated polymers-reference force-field and transferable coupling-constants for polyacetylene. J. Chem. Phys. 98, 7459-7465 (1993).

22. Girlando, A., Painelli, A. \& Soos, Z. G. Towards a unified view of electronphonon coupling in 1d solids. Acta Phys. Pol. A 87, 735-742 (1995).

23. Ferrari, A. C., Rodil, S. E. \& Robertson, J. Interpretation of infrared and raman spectra of amorphous carbon nitrides. Phys. Rev. B 67, 155306 (2003).

24. Peticolas, W. L., Nafie, L., Stein, P. \& Fanconi, B. Quantum theory of intensities of molecular vibrational spectra. J. Chem. Phys. 52, 1576-1584 (1970).

25. Nafie, L. A. Theory of vibrational circular dichroism and infrared absorption: extension to molecules with low-lying excited electronic states. J. Phys. Chem. A 108, 7222-7231 (2004).

26. Zaikowski, L. et al. Polarons, bipolarons, and side-by-side polarons in reduction of oligofluorenes. J. Am. Chem. Soc. 134, 10852-10863 (2012).

27. Takeda, N., Asaoka, S. \& Miller, J. R. Nature and energies of electrons and holes in a conjugated polymer, polyfluorene. J. Am. Chem. Soc. 128, 16073-16082 (2006).
28. Knibbe, H., Rehm, D. \& Weller, A. Intermediates and kinetics of fluorescence quenching by electron transfer. Ber. Bunsenges. Phys. Chem. 72, 257-263 (1968).

29. Takeda, N. \& Miller, J. R. Poly(3-Decylthiophene) radical anions and cations in solution: single and multiple polarons and their delocalization lengths in conjugated polymers. J. Phys. Chem. B 116, 14715-14723 (2012).

30. Nakanishi, K. \& Solomon, P. H. Infrared Absorption Spectroscopy (Emerson Adams Press Inc., 1998).

31. Wilson, E. B., Decius, J. C. \& Cross, P. C. Molecular Vibrations (McGraw-Hill, 1955).

32. Crawford, B. Vibrational Intensities.2. The Use of Isotopes. J. Chem. Phys. 20 977-981 (1952).

33. Crawford, B. Vibrational Intensities.10. Integration Theorems. J. Chem. Phys. 29, 1042-1045 (1958).

34. Decius, J. C. \& Mast, G. B. Atomic polar tensors and effective charge-charge flux model of infrared intensities. J. Mol. Spectrosc. 70, 294-306 (1978).

35. Rice, M. J., Duke, C. B. \& Lipari, N. O. Intramolecular vibrational stabilization of charge-density wave state in organic metals. Solid State Commun. 17, 1089-1093 (1975).

36. Rice, M. J. Organic linear conductors as systems for study of electron-phonon interactions in organic solid-state. Phys. Rev. Lett. 37, 36-39 (1976).

37. Mele, E. J. \& Rice, M. J. Vibrational excitations of charged solitons in polyacetylene. Phys. Rev. Lett. 45, 926-929 (1980).

38. Etemad, S. et al. Infrared-active vibrational-modes of charged solitons in (Ch)X and (Cd)X. Phys. Rev. B 23, 5137-5141 (1981).

39. Ehrenfreund, E., Vardeny, Z., Brafman, O. \& Horovitz, B. Amplitude and phase modes in transpolyacetylene-resonant raman-scattering and induced infrared activity. Phys. Rev. B 36, 1535-1553 (1987).

40. Szwarc, M. \& Lagur-Grodzinski, J. In Ions and Ion Pairs in Organic Reactions (Wiley, 1974).

41. Girlando, A. Charge sensitive vibrations and electron-molecular vibration coupling in bis(ethylenedithio)-tetrathiafulvalene (Bedt-Ttf). J. Phys. Chem. C 115, 19371-19378 (2011).

42. Sreearunothai, P., Asaoka, S., Cook, A. R. \& Miller, J. R. Length and timedependent rates in diffusion-controlled reactions with conjugated polymers J. Phys. Chem. A 113, 2786-2795 (2009).

43. Frisch, M. J. et al. Gaussian 09 (Gaussian Inc., 2009)

44. Henderson, T. M., Janesko, B. G. \& Scuseria, G. E. Generalized gradient approximation model exchange holes for range-separated hybrids. J. Chem. Phys. 128, 194105 (2008).

\section{Acknowledgements}

This research was carried out at Brookhaven National Laboratory under contract DE-AC02-98CH10886 with the US Department of Energy and supported by its Division of Chemical Sciences, Geosciences \& Biosciences, Office of Basic Energy Sciences, and to Laboratory Directed Research Grant number 02544 at Brookhaven National Laboratory for support to M.Z. and partial support to J.R.M. We thank Dr. Andrew Cook for technical assistance and Larry Nafie for discussions.

\section{Authors contributions}

J.R.M. directed this work. S.A. synthesized oligofluorenes and polyfluorene. M.Z. performed the chemical oxidations/reductions and recorded the IR spectra. D.C.G. provided expertise on the acquisition of IR spectra of air sensitive species. All co-authors discussed the results and the interpretation and participated in the manuscript preparation.

\section{Additional information}

Supplementary Information accompanies this paper at http://www.nature.com/ naturecommunications

Competing financial interests: The authors declare no competing financial interests.

Reprints and permission information is available online at http://npg.nature.com/ reprintsandpermissions/

How to cite this article: Zamadar, M. et al. Giant infrared absorption bands of electron and holes in conjugated molecules. Nat. Commun. 4:2818 doi: 10.1038/ncomms3818 (2013). 